\title{
Prediction of falls among older people in residential care facilities by the Downton index
}

\author{
Erik Rosendahl, Lillemor Lundin-Olsson, Kristina Kallin, Jane Jensen, Yngve Gustafson, and \\ Lars Nyberg \\ Department of Community Medicine and Rehabilitation, Geriatric Medicine and Physiotherapy, Umeå \\ University, Umeå, Sweden
}

\begin{abstract}
Background and aims: Falls are frequent among older people living in residential care facilities. The aim of this study was to investigate the prediction accuracy of the Downton fall risk index among older people living in residential care facilities at 3, 6 and 12 months, and with two different definitions of falls. Methods: Seventy-eight residents in one residential care facility, 56 women and 22 men, mean $\pm S D$ age $81 \pm 6$ years, participated in this study. Forty-seven percent of participants had dementia, $45 \%$ depression, and $32 \%$ previous stroke. Forty-one percent of participants used a walking device indoors, and the median score of the Barthel ADL Index was 16. At baseline, the Downton fall risk index was scored for each individual. A score of 3 or more was taken to indicate high risk of falls. Participants were followed up prospectively for 12 months, with regard to falls indoors. Results: At 3, 6 and 12 months, and using a fall definition including all indoor falls, sensitivity ranged from 81 to $95 \%$ with the highest value at 3 months, and specificity ranged from 35 to $40 \%$. The prognostic separation values ranged from 0.26 to 0.37 . Within 3 months, the risk of falling was $36 \%$ in the high-risk group (index score $\geq 3$ ) and $5 \%$ in the low-risk group. The accuracy of predictions did not improve when applying a fall definition in which falls precipitated by acute illness, acute disease, or drug side-effects were excluded. Conclusions: Already after 3 months, the Downton fall risk index appears to be a useful tool for predicting falls, irrespective of their cause, among older people in residential care facilities.
\end{abstract}

(Aging Clin Exp Res 2003; 15: 142-147)

${ }^{\circ} 2003$, Editrice Kurtis

\section{INTRODUCTION}

As falls are frequent among older people living in residential care facilities $(1,2)$, and these people also run a high risk of sustaining hip fracture $(3,4)$, it seems a matter of urgency to find ways of preventing falls.

Identification of high-risk individuals is often seen as an important part of prevention programs. Risk factors discriminating fall-prone individuals from those less prone to fall have been studied extensively. Mobility problems, sensory deficits, cognitive impairment, and the use of psychoactive medications are commonly suggested as important risk factors (5).

One assumption regarding prevention of falls is that the cumulative effect of multiple risk factors contributes more to the tendency to fall than the potential effect of each risk factor alone (6). On the basis of this assumption, a number of fall risk scoring systems screening for well-established risk factors have been presented (7-17). Only two of these, the Tinetti fall risk index and the Mobility Interaction Fall chart, have been developed among elderly people in residential care facilities $(10,17)$. The Tinetti fall risk index seems too complex to be convenient in clinical practice and, to our knowledge, only part of the index, the Tinetti balance scale, has been validated externally (18). There are no reports of validation of the Mobility Interaction Fall chart in an independent sample.

The Downton fall risk index includes well-documented risk factors for falls and therefore offers satisfactory content validity (7), and also seems to be very easy to administer. Although the Downton index was developed for elderly people in continuing care wards, we believe it may also be useful in residential care facilities. Downton (7) presented a moderate association between index score and number of patients with falls during the previous year in a sample of 28 patients. Sensitivity was very high (100\%)

Key words: Accidental falls, fall risk index, older people, prediction accuracy, prevention of falls, residential care.

Correspondence: E. Rosendahl, R.P.T., Department of Community Medicine and Rehabilitation, Geriatric Medicine, Umeå University, SE90187 Umeå, Sweden.

E-mail: erik.rosendahl@germed.umu.se

Received July 11, 2001; accepted in revised form October 29, 2002. 
Table 1 - Characteristics of participants with and without falls during 12 months.

\begin{tabular}{|c|c|c|c|c|}
\hline & Total sample $(\mathrm{N}=78)$ & Without falls $(\mathrm{N}=30)$ & With falls $(\mathrm{N}=48)$ & $p$ \\
\hline Age (years), mean $\pm \mathrm{SD}$ & $81 \pm 6$ & $79 \pm 6$ & $83 \pm 6$ & 0.010 \\
\hline Female sex, \% & 72 & 70 & 73 & 0.781 \\
\hline Dementia, \% & 47 & 37 & 54 & 0.132 \\
\hline Depression, \% & 45 & 17 & 63 & $<0.001$ \\
\hline Previous stroke, $\%$ & 32 & 27 & 35 & 0.420 \\
\hline Use of walking device indoors, \% & 41 & 36 & 45 & 0.446 \\
\hline Living in private rooms, $\%$ & 68 & 53 & 77 & 0.029 \\
\hline $\begin{array}{l}\text { MMSE*, median score } \\
\text { (interquartile range) }\end{array}$ & $\begin{array}{c}21 \\
(12-26)\end{array}$ & $\begin{array}{c}24 \\
(15-28)\end{array}$ & $\begin{array}{c}20 \\
(10-25)\end{array}$ & 0.045 \\
\hline $\begin{array}{l}\text { Barthel ADL index" } \text {, median score } \\
\text { (interquartile range) }\end{array}$ & $\begin{array}{c}16 \\
(11-18)\end{array}$ & $\begin{array}{c}16 \\
(14-19)\end{array}$ & $\begin{array}{c}15 \\
(10-18)\end{array}$ & 0.229 \\
\hline
\end{tabular}

"Mini-Mental State Examination (23); "Barthel Index of ADL (25).

but specificity very low (9\%). To our knowledge, the Downton fall risk index has been validated externally only among stroke patients in geriatric rehabilitation, in which a moderately high correlation was found between predicted risk of falls and observed falls (19).

Follow-up periods vary in studies of fall risk scoring systems, from one day or one week up to six months (10, $12,14)$. However, how the length of the follow-up period affects prediction accuracy is not yet known. Likewise, it may be of importance to consider which definition of a fall to use when applying a fall risk index. The Kellogg International Group on the Prevention of Falls formulated a definition of falls which considers the causes of a fall (20). A fall is defined as "an event which results in a person coming to rest inadvertently on the ground or other lower level and other than as a consequence of the following: sustaining a violent blow, loss of consciousness, sudden onset of paralysis, as in stroke, and an epileptic seizure". Among frail elderly individuals, acute illness, acute disease, and drug side-effects cause many falls (17, 21,22 ) which may be difficult to predict using a fall risk index. We therefore assumed that prediction accuracy may improve when excluding from analyses falls precipitated by acute illness, acute disease, or drug side-effects.

The purpose of our study was to investigate the prediction accuracy of the Downton fall risk index among older people living in residential care facilities at 3, 6 and 12 months, and with two different definitions of falls.

\section{METHODS}

\section{Participants and setting}

The study was performed at a residential care facility in Umeå, Sweden. All residents, aged 65 years and over $(\mathrm{N}=78)$ who were living at the facility in February 1994 or who moved in during the following one-year period were given written and oral information about the study. All of them, or the relatives of residents with severe cognitive dysfunction, gave their informed consent to participation in the study. Subject characteristics are presented in Table 1. The study was approved by the Ethical Committee of the Faculty of Medicine at Umeå University.

All 78 participants had 24-hour daily access to assistance with activities of daily living, household issues, and medical care. Fifty-three participants lived in private rooms but shared dining and living rooms, and 25 participants lived in private apartments. No kind of physical restraint was prescribed.

\section{Baseline assessment procedures}

A physician (YG, KK) registered diagnoses and medications and assessed participants' cognitive function using the Mini-Mental State Examination (MMSE) (23). Depression and dementia were diagnosed using DSM-III-R criteria (24). A registered nurse, employed at the facility and also working part-time on this project, scored activities of daily living (ADL) according to the Barthel ADL Index (25).

\section{Scoring of Downton fall risk index}

The Downton fall risk index (Table 2) includes 11 risk items, which are scored one point each. Scores are summed to a total index score, range $0-11$. A score of 3 or more is taken to indicate a high risk of falls. No explicit operational definitions are provided with the Downton fall risk index, and therefore we specified the definitions used in this study.

A physician (YG, KK) made almost all the assessments for the index. Histories of falls during the preceding year were obtained from medical records, the participants themselves, or family members or caregivers. Medications were grouped according to the Downton index categories. Visual impairment was noted if the participant, with or without glasses, was not able to read a word in 5$\mathrm{mm}$ block letters at reading distance. Hearing impairment was noted if the participant, without a hearing aid, was not able to perceive a conversation in a normal voice 
at a distance of 1 meter. Limb impairment was assessed by a physiotherapist (LLO, LN) and defined as the presence of amputated limb, signs of extremity paresis, muscle weakness or sensory impairment. Regarding mental state, we preferred the term cognitively impaired to confused. We used the well-established MMSE cut-off score of $\leq 23 / 30$ points as a diagnostic criterion indicating cognitively impaired, instead of the suggested cut-off score of $<7 / 10$ points of the Abbreviated Mental Test score (26).

Participants' ability to walk safely was rated by a physiotherapist (LLO, LN) according to the following categories: normal (safe without walking aids), safe with walking aids, unsafe, unable. Safe gait was scored when the participant was able to move easily and safely when, for example, opening and closing doors, meeting people in the hallway, and approaching a chair to sit down. Unsafe gait indicated that the participant moved in an uncontrolled way, staggered or stumbled. For each participant, the score was made by a physiotherapist (LLO, LN). Other coworkers in the study and staff were blinded for total scores.

Follow-up for falls

Participants were followed up prospectively regarding

Table 2 - The Downton fall risk index*.

\begin{tabular}{lc}
\hline Items & Score\# \\
\hline Known previous falls & \\
No & 0 \\
Yes & 1 \\
Medications & \\
None & 0 \\
Tranquillizers/sedatives & 1 \\
Diuretics & 1 \\
Antihypertensives (other than diuretics) & 1 \\
Antiparkinsonian drugs & 1 \\
Antidepressants & 1 \\
Other medications & 0 \\
Sensory deficits & \\
None & 0 \\
Visual impairment & 1 \\
Hearing impairment & 1 \\
Limb impairment & 1 \\
Mental state & \\
Orientated & 0 \\
Confused (cognitively impaired) & 1 \\
Gait & \\
Normal (safe without walking aids) & 0 \\
Safe with walking aids & 0 \\
Unsafe (with/without walking aids) & 1 \\
Unable & 0 \\
\hline
\end{tabular}

"Item scores are added together to an index total, range $0-11$, where 3 or more is taken to indicate a high risk of falls.

\#Downton, 1993 (7). indoor falls at the residential facility for a total period of 12 subsequent months from inclusion in the study, or until they moved or died. The number of observation days was calculated for each individual at 3,6 and 12 months after inclusion in the study. Participants' absence from the facility (if lasting more than two days), in all 319 days among 20 participants, was subtracted from each participant's observation time. The total number of observation days at the 12 -month follow-up was 24536 . The follow-up period for each resident ranged from 12 to 365 days (interquartile range 333-365), and 56\% of the participants had a full one-year follow-up period.

\section{Fall definitions and registration}

Two different definitions of falls were used: 1) an indoor event in which the resident unintentionally came to rest on the floor regardless of whether or not an injury was sustained; and 2) when falls were precipitated by acute illness, acute disease, or drug side-effects, they were excluded.

The staff received brief information about fall registration and the importance of reporting all falls that came to their knowledge. They registered falls on a form and reported each incident to the study nurse, who immediately followed up each fall. The study nurse also supervised and encouraged staff to report falls as accurately as possible. One of the authors (YG) was the geriatrician responsible for the residential care facility and, together with the study nurse, as soon as possible or at least within a few days, he followed up each fall with regard to any injury and evaluated possible precipitating causes of the fall. Acute illness was regarded as a precipitating factor when the resident, before the fall, showed symptoms of illness such as impaired balance or delirium, and when the symptoms disappeared when the illness was treated. Acute disease was regarded as a precipitating factor when a stroke or cardiac infarction was discovered in connection with the fall. A drug was judged to have precipitated the fall when there were reports of sideeffects from a recently prescribed drug and the symptoms disappeared after discontinuation of treatment.

\section{Statistical analysis}

Sensitivity, specificity, and positive and negative predictive values, using both fall definitions, were calculated at 3 , 6, and 12 months after inclusion in the study (all participants included at each time-point). At the same time-points, the Prognostic Separation index (PSEP), as suggested by Altman and Royston (27), was calculated. In short, PSEP is the difference between the probabilities of an event occurring in the group with the worst predicted prognosis, and in the group with the best. The optimal value is 1.0. For all proportions, 95\% CI were calculated, using binomial distribution.

Further, the time to first fall (event-free time) was calculated as the number of observation days until the first fall (if any). The association between the time to first fall (dependent variable) and the total Downton fall risk index 
Table 3 - Accuracy of the Downton fall risk index, with two different fall definitions, during 3, 6 and 12 months.

\begin{tabular}{|c|c|c|c|c|c|c|}
\hline & \multicolumn{3}{|c|}{ All falls included } & \multicolumn{3}{|c|}{$\begin{array}{l}\text { Falls not precipitated by acute illness, } \\
\text { acute disease, or drug side-effects }\end{array}$} \\
\hline & 3 months & 6 months & 12 months & 3 months & 6 months & 12 months \\
\hline Sensitivity, \% (95\% CI) & $95(76-100)$ & $91(75-98)$ & $81(67-91)$ & $100(77-100)$ & $91(72-99)$ & $77(60-90)$ \\
\hline Specificity, \% (95\% CI) & 35 (23-49) & $39(25-55)$ & $40(23-59)$ & $33(22-46)$ & $35(22-49)$ & $30(17-46)$ \\
\hline $\begin{array}{l}\text { Positive predictive } \\
\text { value, } \%(95 \% \mathrm{CI})\end{array}$ & $35(23-49)$ & $51(37-64)$ & $68(55-80)$ & $25(14-38)$ & $37(24-51)$ & 47 (34-61) \\
\hline $\begin{array}{l}\text { Negative predictive } \\
\text { value, \% (95\% CI) }\end{array}$ & $95(76-100)$ & $86(64-97)$ & $57(34-78)$ & $100(84-100)$ & 90 (70-99) & $62(38-82)$ \\
\hline PSEP value (95\% CI) & $0.30(0.08-0.52)$ & $0.37(0.12-0.61)$ & $0.26(0.01-0.50)$ & $0.25(0.05-0.44)$ & $0.27(0.04-0.50)$ & $0.09(-0.16-0.34)$ \\
\hline
\end{tabular}

PSEP: Prognostic Separation index (range 0-1.0).

score (independent variable) was analyzed using the Cox regression, with calculation of the hazard ratio (HR). The same analyses were also performed applying the allocation to high or low fall risk groups, and separate item scores respectively as independent variables. Kaplan-Meier analysis with the Log Rank test for statistical significance was also used.

Statistical analyses were computed using the SPSS software package (28). A $p$-value $<0.05$ was considered statistically significant.

\section{RESULTS}

A total of 148 indoor falls occurred during the 12month period, corresponding to an event rate of 2.2 (95\% CI 1.9-2.6) falls per person per year. Of the residents, 48 out of $78(62 \%)$ suffered at least one fall, and $30(38 \%)$ fell twice or more. The number of falls per person ranged from 0 to 22 .

Thirty-two falls were regarded as being precipitated by acute illness, 8 by acute disease, 12 by drug side-effects, and two falls were regarded as precipitated by both acute illness and drug side-effects. When all these falls were excluded to fit the second definition of falls, a total of 94 falls remained among 35 residents.

The median score on the Downton index was 4 (interquartile range $2-5$, range $0-9)$. Fifty-seven $(73 \%)$ participants scored 3 or more on the index, thus reaching the suggested cut-off score for high risk of falls.

As Table 3 shows, with all falls included, sensitivity ranged from 81 to $95 \%$, with the highest value at 3 months, while specificity ranged from 35 to $40 \%$. PSEP ranged from 0.26 to 0.37 at the three different timepoints. The highest positive predictive value was $68 \%(12$ months) and the highest negative predictive value was 95\% (3 months). When excluding falls precipitated by acute illness, acute disease, or drug side-effects, sensitivity was $100 \%$ at 3 months, decreasing to $77 \%$ at 12 months. PSEP ranged from 0.09 to 0.27 , the value at 12 months being statistically insignificant.

The time to first fall differed significantly between low- and high-risk groups (Fig. 1). The risk of falling within 3 months was $36 \%$ in the high-risk group and $5 \%$ in the lowrisk group. Within 12 months, the risk was 76 and $47 \%$, respectively. The Hazard Ratio (HR) was 2.5 (95\% CI 1.2$5.2, p=0.012)$ with all falls included and 2.3 (95\% CI 1.1$4.8, p=0.022$ ) when excluding falls precipitated by acute illness, acute disease, or drug side-effects.

In an analysis of the total index score, the sum of the risk items was also significantly associated with the time to first fall; HR for the total score with all falls included was 1.5 (95\% CI 1.2-1.7, $p<0.001$ ) and, when using the second definition of falls, it was 1.4 (95\% CI 1.1-1.6, $p=0.002)$.

As Table 1 shows, age, depression, living in private rooms (compared with private apartments) and MMSE scores were significantly associated with falling. However, the associations between time to first fall and highor low-risk group allocation remained significant when adjusting for age, sex, and living accommodation (data not

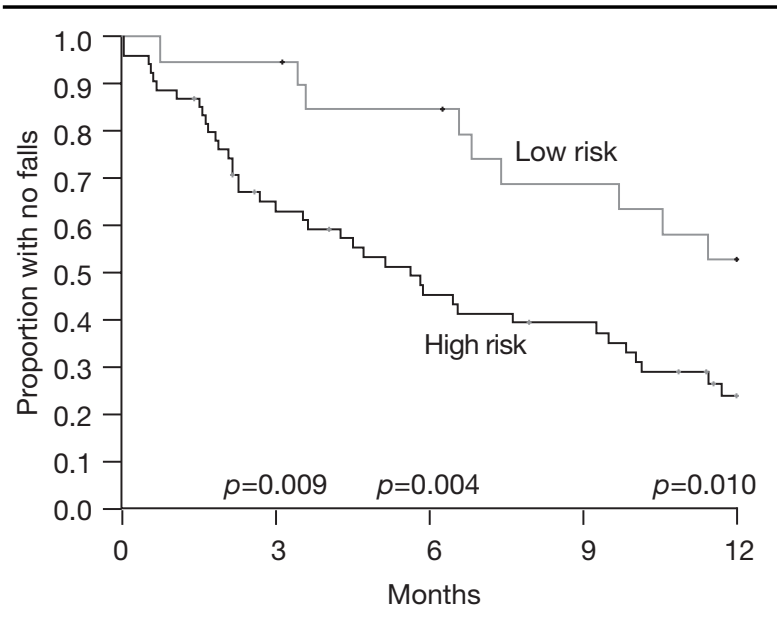

Figure 1 - Kaplan-Meier curves of time to first fall among participants in high-risk $(N=57)$ and low-risk $(N=21)$ groups. $p$-values refer to Log Rank tests at 3, 6 and 12 months, respectively. 
Table 4 - Association between separate Downton fall risk index items and time to first fall during 12 months.

\begin{tabular}{|c|c|c|c|c|c|}
\hline Risk items & $\begin{array}{c}\text { Participants } \\
\text { without falls } \\
\%(\mathrm{~N}=\mathbf{3 0})\end{array}$ & $\begin{array}{c}\text { Participants } \\
\text { with falls } \\
\%(\mathrm{~N}=48)\end{array}$ & HR & $95 \% \mathrm{CI}$ & $p$ \\
\hline Known previous falls & 33 & 56 & 1.67 & $0.94-2.95$ & 0.080 \\
\hline Tranquillizers/sedatives & 43 & 58 & 1.66 & $0.93-2.96$ & 0.085 \\
\hline Diuretics & 43 & 42 & 1.15 & $0.65-2.05$ & 0.627 \\
\hline Antihypertensives (other than diuretics) & 10 & 12 & 1.22 & $0.52-2.88$ & 0.647 \\
\hline Antiparkinsonian drugs & 3 & 8 & 1.45 & $0.52-4.05$ & 0.481 \\
\hline Antidepressants & 10 & 33 & 1.93 & $1.05-3.52$ & 0.033 \\
\hline All medication items $(0-5)$ & - & - & 1.36 & $1.04-1.78$ & 0.027 \\
\hline Visual impairment & 7 & 31 & 3.14 & $1.69-5.84$ & $<0.001$ \\
\hline Hearing impairment & 30 & 38 & 1.61 & $0.90-2.89$ & 0.110 \\
\hline Limb impairment & 33 & 38 & 1.04 & $0.58-1.87$ & 0.891 \\
\hline All sensory deficit items (0-3) & - & - & 1.54 & $1.11-2.13$ & 0.009 \\
\hline Cognitively impaired & 47 & 71 & 2.27 & $1.21-4.25$ & 0.011 \\
\hline Unsafe gait & 30 & 50 & 1.34 & $1.11-1.63$ & 0.003 \\
\hline
\end{tabular}

shown). Since depression and MMSE scores were parts of, or closely related to parts of the index, no adjustments were made for these variables.

Table 4 describes the associations of separate risk items with the time to first fall. As may be seen, use of antidepressants, visual impairment, cognitively impaired, and unsafe gait were separately associated with the fall risk. The items: previous falls, use of tranquillizers/sedatives and hearing impairment were close to statistical significance when analyzed as separate factors, while the other medication items and limb impairment were far from significantly associated. Still, when added together in groups, both medication and sensory deficit items were significantly associated with the time to first fall.

\section{DISCUSSION}

The Downton fall risk index seems to be a useful tool in predicting the risk of falls among older people in residential care. At 3 months, more than one out of 3 in the high-risk group had suffered a fall, compared with only one in 20 in the low-risk group.

This study indicates a predictive accuracy at least on the same level as other external validations of fall risk indexes. Validation of the Downton index among stroke patients in geriatric rehabilitation, follow-up time range 3289 days, showed a sensitivity of $91 \%$ and a specificity of $27 \%$ (19). In an external validation of the Tinetti balance scale, part of the Tinetti fall risk index, with a 12-month follow-up among community-dwelling people, the most accurate cut-off score resulted in a sensitivity of $70 \%$ and a specificity of $52 \%$ (18).

In the present study, the Downton fall risk index showed the highest sensitivity at 3 months. The highest PSEP value was found at 6 months, but a statistically significant prognostic separation, almost as wide, was already seen at 3 months. It is probable that changes in health status which occur during one year have an effect on the fall risk and also on the index score among frail older people in residential care, and it may be recommended to screen for fall risk every third month.

To our surprise, the Downton fall risk index did not show better prediction accuracy when falls judged as precipitated by acute illness, acute disease, and side-effects from a recently prescribed drug were excluded. It may be that the Downton index marks frailty and thus susceptibility to these precipitating factors for falls. The results of our study indicate that the Downton index predicts falls irrespective of their cause.

Three of the medication items and one of the sensory deficit items showed a weak association with the fall risk, and it may be disputed whether they make any significant contribution to fall prediction. In the literature, two meta-analyses call in question whether there is a strong association between analgesic, cardiac and psychotropic drugs, and falls $(29,30)$. Five of eleven risk items of the Downton index reflect medication as a predisposing factor, which may be seen as an overestimation of medication as an important fall risk factor. Instead, when medication items were added together to form a sub-score of the index, they were significantly associated with the fall risk. This may reflect the fact that the combination of different drugs contributes more to the fall risk than what is mediated by each type of drug by itself (30).

In our view, sensitivity is a highly significant quality of a fall prediction instrument, especially if the prediction forms the basis for recruiting individuals in preventive programs. The high sensitivity of the Downton index shown in our study confirms that relatively few individuals are falsely predicted as being at low risk of falling. The low specificity of the index is more acceptable than low sen- 
sitivity would be, as long as the fall intervention does not lead to any harm to the individual, for example through the use of physical restraints.

The Downton index is easy to administer and includes many items that can be obtained from medical records. We believe it can be used routinely in residential care facilities, especially when some items are already made as routine assessments.

The fact that a nurse working at the facility was also employed part-time on this project should contribute to the accuracy of fall reporting, although it was impossible to obtain information about every single fall in this frail and cognitively impaired population. However, a larger sample from more than one single facility would have increased the generalizability of this study. An interrater reliability study would also be of great value.

\section{CONCLUSIONS}

Already after 3 months, the Downton fall risk index appears to be a useful tool for predicting falls among older people in residential care facilities. In comparison with a fall definition with all indoor falls included, a definition in which falls precipitated by acute illness, acute disease, or drug side-effects were excluded did not improve the accuracy of fall prediction.

\section{ACKNOWLEDGEMENTS}

The authors are grateful to K. Strandfur-Byström for her major contribution to data collection. This work was supported by grants from the County Council of Västerbotten, the Federation of County Councils in Sweden, the Umeå University Foundation for Medical Research, Gun and Bertil Stohnes Foundation, and the Swedish Foundation for Health Care Sciences and Allergy Research.

\section{REFERENCES}

1. Luukinen H, Koski K, Hiltunen L, Kivelä SL. Incidence rate of falls in an aged population in northern Finland. J Clin Epidemiol 1994; 47: 843-50.

2. Jensen J, Lundin-Olsson L, Nyberg L, Gustafson Y. Falls among frail older people in residential care. Scand J Public Health 2002; 30: 54-61.

3. Butler M, Norton R, Lee-Joe T, Cheng A, Campbell AJ. The risks of hip fracture in older people from private homes and institutions. Age Ageing 1996; 25: 381-5.

4. Ramnemark A, Nilsson M, Borssén B, Gustafson Y. Stroke, a major and increasing risk factor for femoral neck fracture. Stroke 2000; 31: 1572-7.

5. Myers AH, Young Y, Langlois JA. Prevention of falls in the elderly. Bone 1996; 18: 87S-101S.

6. Tinetti ME, Speechley M. Prevention of falls among the elderly. N Engl J Med 1989; 320: 1055-9.

7. Downton JH. Falls in the elderly. London: Edward Arnold, 1993: $128-30$.

8. Hendrich A, Nyhuis A, Kippenbrock T, Soja ME. Hospital falls: development of a predictive model for clinical practice. Appl Nurs Res 1995; 8: 129-39.

9. Heslin K, Towers J, Leckie C, et al. Managing falls: Identifying population-specific factors and prevention strategies. In: Funk SG,
Tornquist EM, Champagne MT, Wiese RA, Eds. Key aspects of elder care: managing falls, incontinence, and cognitive impairment. New York: Springer Publ. Co, 1992: 70-88.

10. Lundin-Olsson L, Nyberg L, Gustafson Y. The Mobility Interaction Fall chart. Physiother Res Int 2000; 5: 190-201.

11. MacAvoy S, Skinner T, Hines M. Fall risk assessment tool. Appl Nurs Res 1996; 9: 213-8.

12. Morse JM, Morse RM, Tylko SJ. Development of a scale to identify the fall-prone patient. Can J Aging 1989; 8: 366-77.

13. Nyberg L, Gustafson Y. Fall prediction index for patients in stroke rehabilitation. Stroke 1997; 28: 716-21.

14. Oliver D, Britton M, Seed P, Martin FC, Hopper AH. Development and evaluation of evidence based risk assessment tool (STRATIFY) to predict which elderly inpatients will fall: case-control and cohort studies. BMJ 1997; 315: 1049-53.

15. Schmid NA. 1989 Federal Nursing Service Award Winner. Reducing patient falls: a research-based comprehensive fall prevention program. Mil Med 1990; 155: 202-7.

16. Studenski S, Duncan PW, Chandler J, et al. Predicting falls: the role of mobility and nonphysical factors. J Am Geriatr Soc 1994; 42: 297-302.

17. Tinetti ME, Williams TF, Mayewski R. Fall risk index for elderly patients based on a number of chronic disabilities. Am J Med 1986; 80: 429-34.

18. Raiche M, Hebert R, Prince F, Corriveau H. Screening older adults at risk of falling with the Tinetti balance scale. Lancet 2000; 356: 1001-2.

19. Nyberg L, Gustafson Y. Using the Downton index to predict those prone to falls in stroke rehabilitation. Stroke 1996; 27 : 1821-4.

20. The prevention of falls in later life. A report of the Kellogg International Work Group on the Prevention of Falls by the Elderly. Dan Med Bull 1987; 34 (Suppl 4): 1-24.

21. Nurmi I, Sihvonen M, Kataja M, Luthje P. Falls among institutionalized elderly. A prospective study in four institutions in Finland. Scand J Caring Sci 1996; 10: 212-20.

22. Kallin K, Lundin-Olsson L, Jensen J, Nyberg L, Gustafson Y. Predisposing and precipitating factors for falls among older people in residential care. Public Health 2002; 116: 1-9.

23. Folstein MF, Folstein SE, McHugh PR. Mini-mental state. A practical method for grading the cognitive state of the patient for the clinician. J Psychiatr Res 1975; 12: 189-98.

24. American Psychiatric Association: Diagnostic and Statistical Manual of Mental Disorders (DSM-III-R), ed. 3. Washington DC: American Psychiatric Association Press, 1987: 103-7, 218-35.

25. Mahoney FI, Barthel DW. Functional evaluation: the Barthel Index. Maryland State Med J 1965; 14: 61-5.

26. Qureshi KN, Hodkinson HM. Evaluation of a ten-question mental test in the institutionalized elderly. Age Ageing 1974; 3: 152-7.

27. Altman DG, Royston P. What do we mean by validating a prognostic model? Stat Med 2000; 19: 453-73.

28. Norusis MJ. SPSS advanced statistics 6.1. SPSS Inc., United States of America, 1994.

29. Leipzig RM, Cumming RG, Tinetti ME. Drugs and falls in older people: a systematic review and meta-analysis: I. Psychotropic drugs. J Am Geriatr Soc 1999; 47: 30-9.

30. Leipzig RM, Cumming RG, Tinetti ME. Drugs and falls in older people: a systematic review and meta-analysis: II. Cardiac and analgesic drugs. J Am Geriatr Soc 1999; 47: 40-50. 